\title{
Agradecimiento a los árbitros
}

\section{Agradecemos la participación de los siguientes árbitros:}

Hugo Antonio Arciniega, Universidad Nacional Autónoma de México, México

José Ricardo Gutiérrez Vargas, Instituto Tecnológico de Estudios Superiores de Monterrey, México

Elisa María Teresa Drago Quagua, Universidad Nacional Autónoma de México, México

Irene Gras Valero, Universidad de Barcelona, España

Edwin Culp, Universidad Iberoamericana, México

Samuel Hernández Dominicis, Universidad Iberoamericana, México

Trinidad Pérez Arias, Universidad Andina Simón Bolívar, Ecuador

Santiago Javier Ávila Albuja, Universidad Israel, Ecuador

Yuri Marat Gómez Cervantes, Universidad Nacional Mayor de San Marcos, Perú

Gloria Bonilla Vélez, Universidad de Cartagena, Colombia

Blanca Gutiérrez Galindo, Universidad Nacional Autónoma de México, México

Sandra Zetina Ocaña, Universidad Nacional Autónoma de México, México

Sarelys Avendaño Escobar, Universidad del Atlántico, Colombia 